\title{
SYNAPTIC TRANSFER OF DYNAMIC MOTION INFORMATION BETWEEN IDENTIFIED NEURONS IN THE VISUAL SYSTEM OF THE BLOWFLY
}

\author{
A.-K. WARZECHA, ${ }^{*}$ R. KURTZ AND M. EGELHAAF \\ Lehrstuhl für Neurobiologie, Fakultät für Biologie, Universität Bielefeld, \\ Postfach 1001 31, D-33501 Bielefeld, Germany
}

\begin{abstract}
Synaptic transmission is usually studied in vitro with electrical stimulation replacing the natural input of the system. In contrast, we analyzed in vivo transfer of visual motion information from graded-potential presynaptic to spiking postsynaptic neurons in the fly. Motion in the null direction leads to hyperpolarization of the presynaptic neuron but does not much influence the postsynaptic cell, because its firing rate is already low during rest, giving only little scope for further reductions. In contrast, preferred-direction motion leads to presynaptic depolarizations and increases the postsynaptic spike rate. Signal transfer to the postsynaptic cell is linear and reliable for presynaptic graded membrane potential fluctuations of up to approximately $10 \mathrm{~Hz}$. This frequency range covers the dynamic range of velocities that is encoded with a high gain by visual motionsensitive neurons. Hence, information about preferred-direction motion is transmitted largely undistorted ensuring a consistent dependency of neuronal signals on stimulus parameters, such as motion velocity. Postsynaptic spikes are often elicited by rapid presynaptic spike-like depolarizations which superimpose the graded membrane potential. Although the timing of most of these spike-like depolarizations is set by noise and not by the motion stimulus, it is preserved at the synapse with millisecond precision.
\end{abstract}

Key words: synapse, visual motion, neural computation, reliability, spike timing, graded potentials.

Synapses represent key elements in neuronal information processing, since they often do not merely transmit information. Instead, they may act as temporal filters (Fortune and Rose, 1997, 2001; Trussell, 1999) and may change their properties in an activity-dependent way (Thomson, 2000; Fortune and Rose, 2001). We address the question of how synaptic transfer is tuned to the dynamics of the presynaptic input and of the sensory stimuli processed by the system.

The analysis is done on an identified synaptic connection in the visual motion pathway of the blowfly. At this connection the presynaptic neurons encode visual motion information by graded shifts of their membrane potential with superimposed spike-like depolarizations ('spikelets'), which are based on voltage-dependent sodium conduc-

${ }^{*}$ Corresponding author. Tel: +49-521-106-5578; fax: +49-521-1066095.

E-mail address: ak.warzecha@uni-bielefeld.de (A.-K. Warzecha).

Abbreviations: TC, tangential cell. tances. These may be partly inactivated during pronounced motion-induced depolarisations, resulting in a highly variable spikelet amplitude, ranging from a few millivolts to approximately $40 \mathrm{mV}$ (Hausen, 1982; Haag et al., 1997). Although neurons in various systems show such a mixed response type (e.g. Angstadt and Calabrese, 1993; Manor et al., 1997; Isaacson and Strowbridge, 1998; Simmons, 2002a,b) it is not yet clear how synapses cope with the combination of different modes and timescales in presynaptic activity.

The relevant timescale of signal transfer between neurons can only be assessed if the dynamics of the signals under the system's normal operating conditions are known. Therefore, we investigate synaptic signal transfer under in vivo conditions where the natural range and temporal pattern of synaptic activity can be evoked by sensory stimulation. The analyzed neurons belong to a neuronal ensemble, the tangential cells (TCs), that is tuned to the flow of retinal images as it occurs during locomotion (Hausen and Egelhaaf, 1989; Egelhaaf et al., 2002). Most TCs integrate the signals of many local motion-sensitive elements subserving the ipsilateral eye. Other TCs receive their input from such integrating TCs and convey optic flow information to the contralateral visual system (Hausen and Egelhaaf, 1989; Hausen, 1981; Warzecha et al., 1993; Strausfeld et al., 1995; Horstmann et al., 2000; Krapp et al., 2001; Haag and Borst, 2001). An example of the latter type of TC is the V1-cell that is probed in the present study (Fig. 1A). The V1-cell is postsynaptic to three VS-neurons (for details see below and Kurtz et al., 2001). The receptive fields of the three VS-cells overlap, but their centers are slightly shifted with respect to each other (Krapp et al., 2001). Whereas $V 1$ transmits visual motion information by spikes (Krapp et al., 2001), VS-cells respond to visual motion by graded de- or hyperpolarizations with superimposed spikelets (Fig. 1B, Krapp et al., 1998; Hengstenberg, 1982). Both signal components, i.e. graded membrane potential changes and spikelets, can be recorded close to the output terminal of VS-cells and, therefore, are expected to be present at the terminal membrane. Hence, the presynaptic activity of VS-cells varies on a wide range of timescales and shows a complex dynamic structure.

In a previous account we could show that the graded presynaptic depolarizations of VS-cells induced by constant velocity motion are transformed almost linearly into the postsynaptic spike rate of V1 (Kurtz et al., 2001). While constant velocity stimuli facilitated in this study the imaging of motion-induced changes in presynaptic calcium concentration, it is clear that this type of visual motion hardly ever 

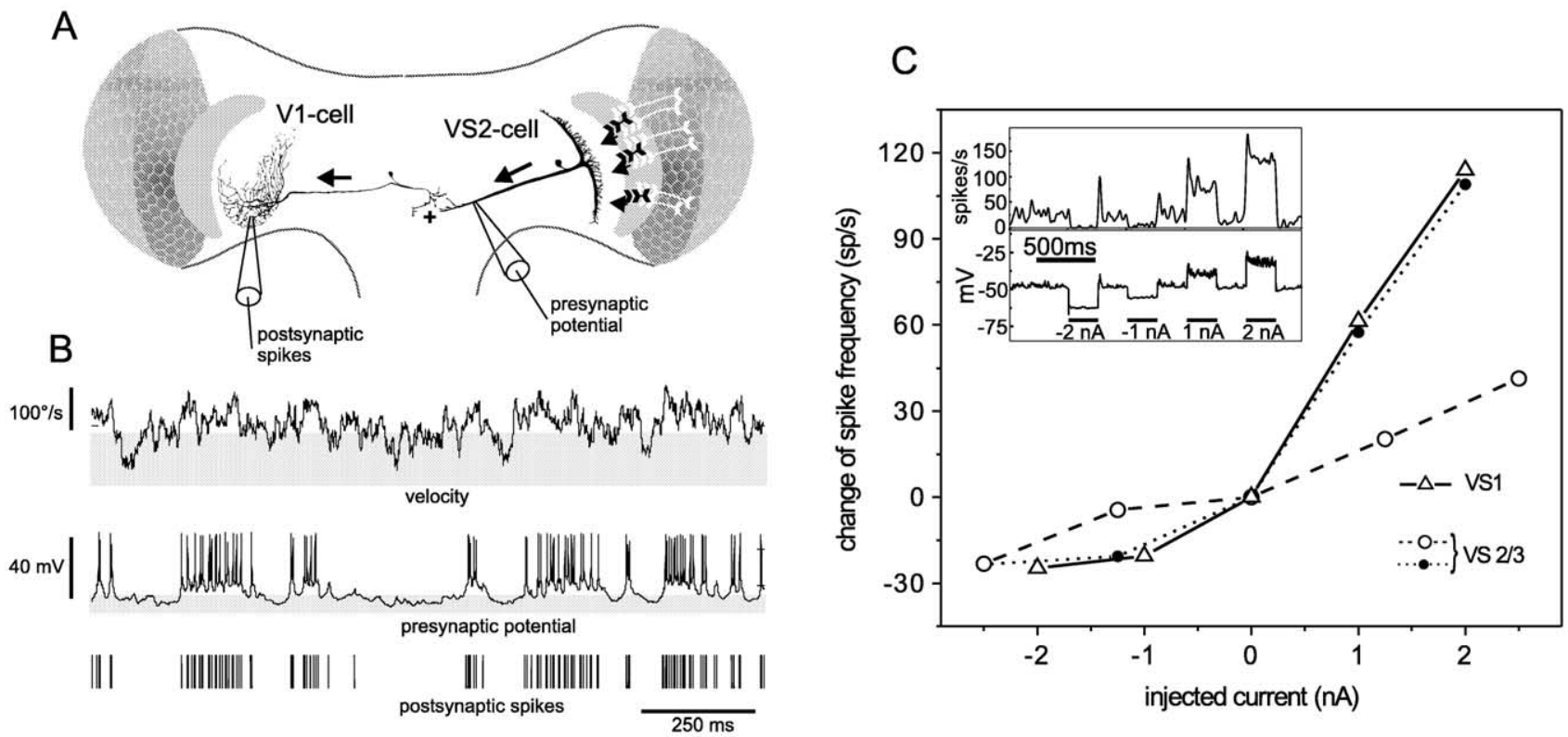

Fig. 1. Synaptic coupling between VS-cells and V1-cell. (A) Schematic of the visual motion pathway of the fly (caudal view) with one of the three presynaptic cells, the VS2-cell, and the postsynaptic V1-cell (reconstructions taken from Hausen and Egelhaaf, 1989). The visual system is organized in a retinotopic way by columnar elements (sketched schematically). The outputs of such small-field motion-sensitive elements are pooled by the large dendrite of the VS2-cell. The signals are conveyed to the axon terminal of the VS2-cell and synaptically transmitted to the V1-cell. Spikes of the V1-cell are propagated to the profusely branched output region in the contralateral visual system. (B) Sample dual recording of the presynaptic potential of the VS1-cell and the postsynaptic spikes of the V1-cell during dynamic motion stimulation (action potentials indicated by vertical lines). (C) Injection of current into the presynaptic VS-cell leads to changes in postsynaptic spike rate of V1. Depolarizing current leads to increases, hyperpolarizing current to decrease in the postsynaptic spike rate. Data from three experiments are shown; the presynaptic cell type is indicated by symbols. Inset: Sample spike frequency histogram of postsynaptic V1-cell (upper trace) and presynaptic potential of VS1-cell (bottom trace) are averages from 10 individual records. The time intervals of current injection and the current amplitudes are indicated below the presynaptic recording.

occurs under natural conditions, where the retinal image is continually displaced while the animal moves around in its environment. Therefore, we extended the analysis to dynamic velocity fluctuations. This dynamic stimulation now allows us to address the following questions. (i) Does linearity of synaptic transfer also hold for dynamic stimulation? (ii) On what timescale do postsynaptic spikes couple to presynaptic potential changes? (iii) What is the relevant timescale for conveying visual motion information?

\section{EXPERIMENTAL PROCEDURES}

\section{Preparation and electrophysiology}

For electrophysiological experiments female blowflies of the genus Calliphora were taken from our laboratory stocks. Dissection of animals followed our standard routine as described elsewhere (see e.g. Dürr and Egelhaaf, 1999). The experiments were performed at room temperature $\left(21-23^{\circ} \mathrm{C}\right)$.

Experiments were started by recording $\mathrm{V} 1$ extracellularly in its output arborizations in the left optic lobe (see Fig. 1). V1 was identified by its sensitivity to downward motion in the frontal part of the right visual field. Extracellular recording was done with standard equipment using glass electrodes (resistance: 4-8 $\mathrm{M} \Omega$ when filled with $1 \mathrm{M} \mathrm{KCl}$ ). Spikes were thresholded and the resulting pulses sampled at $4096 \mathrm{~Hz}$. Once a stable V1 recording was established, a VS-neuron was penetrated close to its output terminal in the right visual system. VS-neurons were identified by graded depolarizations during downward motion in circumscribed parts of the right visual field (Krapp et al., 1998). Intracellular electrodes had resistances of $20-40 \mathrm{M} \Omega$ when filled with $1 \mathrm{M} \mathrm{KCl}$. The membrane potential was analog-digital converted at a rate of $4096 \mathrm{~Hz}$ with an amplitude resolution of $0.0244 \mathrm{mV}$.

\section{Visual stimulation}

Moving square-wave gratings were used as stimuli (spatial wavelength: $18^{\circ}$, mean luminance: $20.5 \mathrm{~cd} / \mathrm{m}^{2}$ ) and displayed on a cathode ray tube (Tektronix 608) at a frame rate of $183 \mathrm{~Hz}$ by a PC-controlled image synthesizer (Picasso, Innisfree, Cambridge, MA, USA). In previous investigations it was ensured that spikes do not time lock to this frame rate (Warzecha and Egelhaaf, 1999; Warzecha et al., 1998). The center of the monitor screen was at an azimuth/elevation of $60^{\circ} / 23^{\circ}$ with $0^{\circ} / 0^{\circ}$ corresponding to the frontal midline of the fly. The frontal edge of the screen had an azimuthal position of $-10^{\circ}$. The horizontal extent of the stimulus pattern was $120^{\circ}$, its vertical extent $104^{\circ}$.

Pattern motion lasted for $4 \mathrm{~s}$. Two types of pattern motion were used: constant and randomly fluctuating velocity. The brightness contrast of the constant velocity stimuli was either $0.14,0.26$ or 0.67 . To obtain a dynamic velocity stimulus, Gaussian whitenoise velocity fluctuations were generated and low-pass filtered with a cutoff at $80 \mathrm{~Hz}$ to avoid aliasing due to the frame rate limit. The S.D. of the final velocity fluctuations was $111 \%$ s. On average, the velocity of the motion stimulus was $0 \%$. Individual stimulus presentations were separated by intervals of $2.5 \mathrm{~s}$ during which the display assumed mean luminance. To attain independence from peculiarities of a particular stimulus, 16 different dynamic stimulus traces with the same statistical properties were presented in pseudo-random order. The contrast of the dynamic stimuli was 0.67 . 
A

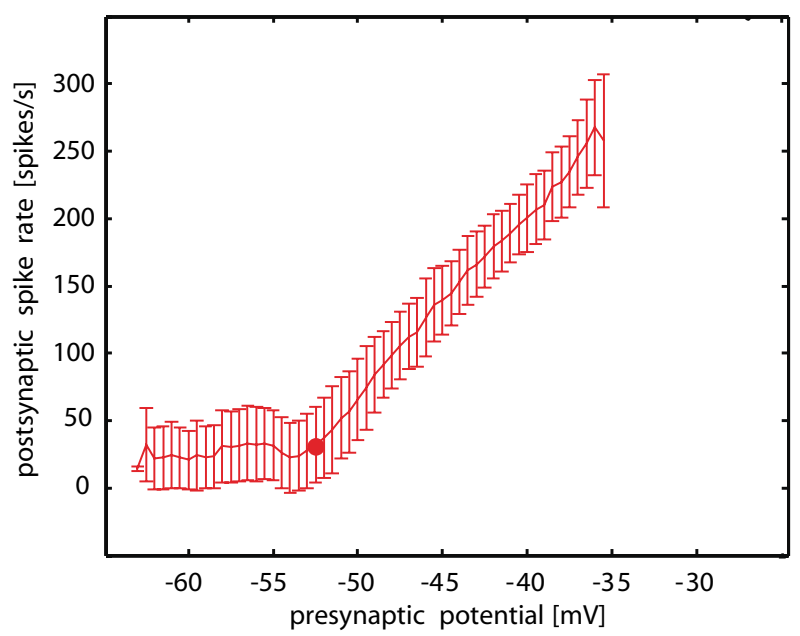

B

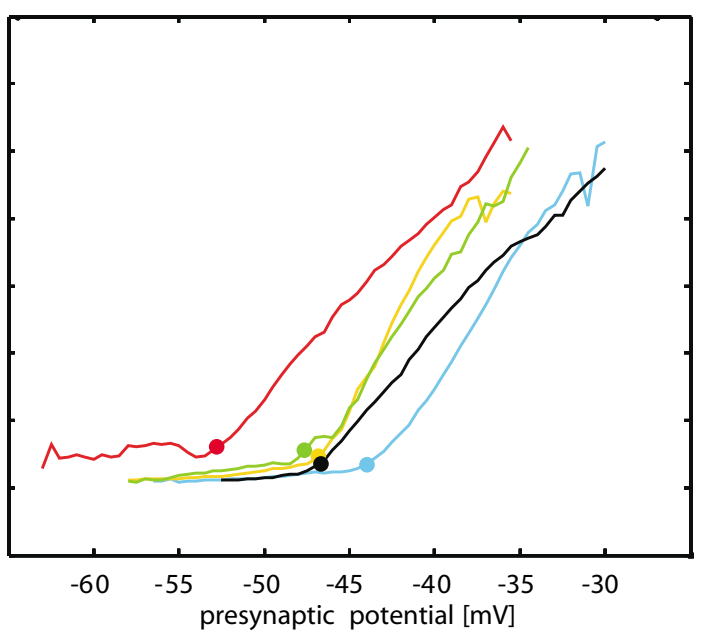

Fig. 2. Relationship between presynaptic potential of VS-cells and postsynaptic spike rate of V1 during stimulation with random-velocity fluctuations. (A) Example from an individual cell pair. The mean and S.D. of the postsynaptic spike rate are plotted as a function of the presynaptic membrane potential. (B) Data from all analyzed cell pairs (S.D.s not shown). Each color denotes the data of a given cell pair. Black indicates the recording with the VS1-cell as presynaptic element. The other colors indicate recordings with VS2/3 as presynaptic elements. This color code will be maintained throughout the following figures. The resting potentials of the VS-cells are indicated by thick dots on the respective data curves. (A, B) The instantaneous spike rate of V1 was estimated from the individual spike trains as the inverse of the corresponding interspike intervals. Both the instantaneous spike rate and the presynaptic potential were smoothed with a Gaussian filter $(\sigma=20 \mathrm{~ms})$. The individually filtered presynaptic potential traces were subdivided into $0.5-\mathrm{mV}$ classes. For each instant of time the postsynaptic spike rate was assigned to the corresponding presynaptic potential class. Finally, the mean and S.D. of the spike rate values were determined for each membrane potential class. Data obtained with all 16 dynamic motion sequences were pooled.

\section{Data analysis}

The data were evaluated off-line with programs written in MatLab 5.0 (The MathWorks, Natick, MA, USA). The transformation of the presynaptic potential into postsynaptic spike activity was analyzed in various ways:

To determine the relationship between pre- and postsynaptic signals (as shown in Fig. 2), the time-dependent 'instantaneous spike rate' was estimated from the individual spike trains as the inverse of the corresponding interspike interval. The spike rate and the presynaptic potential were smoothed with a Gaussian filter $(\sigma=20 \mathrm{~ms})$. The filtered presynaptic potential was subdivided into $0.5-\mathrm{mV}$ classes. For each instant of time the postsynaptic spike rate was assigned to the corresponding presynaptic potential class. The mean and S.D. of the spike rate values were determined for each class and plotted as a function of the presynaptic membrane potential. Data obtained with all 16 dynamic motion traces were pooled. The exact width of the filter is not very critical since we obtained basically the same results for filters with an S.D. of $10 \mathrm{~ms}$ and $40 \mathrm{~ms}$.

Reverse reconstruction was used according to well-established procedures (Dayan and Abbott, 2001; Gabbiani and Koch, 1998) to estimate the presynaptic membrane potential from the postsynaptic spike trains. The VS-cells are depolarized during preferred-direction motion and hyperpolarized to more negative potentials than the resting level during motion in the opposite direction, the so-called null direction. In contrast, the spike activity of $\mathrm{V} 1$ increases during preferred-direction motion, but cannot decrease much during null-direction motion since the resting activity of $\mathrm{V} 1$ is low (Fig. 1B). To account for this rectification, segments of the presynaptic potential that were below the neuron's resting potential were set to the resting potential for reverse reconstruction (see also Machens et al., 2001). In this way it is possible to focus the analysis on the operating range of the V1-cell. The presynaptic $\left(p_{\mathrm{i}}\right)$ and postsynaptic responses $\left(r_{\mathrm{i}}\right)$ to each of the 16 dynamic stimulus traces $\left(s_{i}\right)$ were Fourier-transformed to $P_{\mathrm{i}}(\mathrm{f})$ and $R_{\mathrm{i}}(\mathrm{f})$ and the auto- and cross-correlations were calculated as the products of the complex functions. The frequency response of the reconstruction filter is then given by

$H(\mathrm{f})=\left\langle R_{\mathrm{i}}^{*}(\mathrm{f}) \cdot P_{\mathrm{i}}(\mathrm{f})\right\rangle\left\langle\left\langle R_{\mathrm{i}}(\mathrm{f}) \cdot R_{\mathrm{i}}^{*}(\mathrm{f})\right\rangle\right.$

\langle\rangle denotes the average over the individual responses to all 16 stimuli; - denotes the dot product and * denotes the complex conjugate.

The estimated presynaptic signal was calculated in the time domain by convolving the postsynaptic spike train with the impulse response of the best linear filter obtained by reverse Fourier transformation of $H(\mathrm{f})$. To test to what extent the presynaptic potential is transformed linearly and reliably into the postsynaptic spike activity, the coherence function was determined (Marmarelis and Marmarelis, 1978; Borst and Theunissen, 1999):

$\gamma^{2}(\mathrm{f})=\left(\left\langle R_{\mathrm{i}}{ }^{*}(\mathrm{f}) \cdot P_{\mathrm{i}}(\mathrm{f})\right\rangle \cdot\left\langle P_{\mathrm{i}}{ }^{*}(\mathrm{f}) \cdot R_{\mathrm{i}}(\mathrm{f})\right\rangle\right) /\left(\left\langle P_{\mathrm{i}}(\mathrm{f}) \cdot P_{\mathrm{i}}{ }^{*}(\mathrm{f})\right\rangle \cdot\left\langle R_{\mathrm{i}}(\mathrm{f}) \cdot R_{\mathrm{i}}{ }^{*}(\mathrm{f})\right\rangle\right)$

In a perfectly linear and noise-free system, the coherence function equals one for all frequencies. In a frequency range where the coherence is close to one, the system can be regarded as very reliable and approximately linear. Without rectification of the presynaptic signal the coherence was found to be slightly smaller than is the case when the presynaptic signal is rectified (unpublished results). This reduction is due to the fact that the capacity of the postsynaptic neuron to signal motion in the null direction is restricted to a very small response range compared with the range of responses elicited by motion in the preferred direction (see above). Hence such a reduction of the coherence pertains to spike generation in the postsynaptic neuron rather than to synaptic transmission. Therefore, all analysis presented here was done with rectified presynaptic signals which cover the postsynaptic operating range more adequately than non-rectified signals.

Reverse reconstruction was also employed to determine the best linear filter by which the stimulus can be estimated on the basis of either the presynaptic or the postsynaptic response. For equivalent reasons as outlined above, the rectified forms of the the 
stimulus and the presynaptic response were used for reverse reconstruction.

Correlation techniques were used to analyze the time locking of pre- and postsynaptic responses on a fine timescale. The details are given in the Results section and figure legends.

Although fly TCs are comparatively easy to record from, it is still very hard to do joint recordings from pairs of cells for a sufficiently long time to permit the quantitative analyses that we performed. Moreover, the data had to be carefully checked for drifts and other non-stationarities to avoid artifactual results. Therefore, a large proportion of recordings had to be discarded. The analysis done with white-noise velocity fluctuations is based on one dual recording between VS1 and V1 and on four dual recordings between VS2/3 and V1. The 16 dynamic motion traces were presented in each experiment two to five times. The data obtained with constant-velocity stimuli are based on two dual recordings of VS $2 / 3$ and $\mathrm{V} 1$. In each experiment, the three different contrasts were presented 11 times in pseudorandom order.

Power spectra of the stimulus-induced response components of the presynaptic VS-cell, i.e. the component of the response that is directly linked to the stimulus and free of noise, were calculated as the Fourier transforms of the cross-correlograms of pairs of response traces that were recorded during presentation of the same stimulus trace.

\section{RESULTS}

The synaptic transfer of visual motion information between presynaptic VS-cells and the postsynaptic V1-cell was studied in vivo (for an example see Fig. 1A). V1 receives input from three of the 10 VS-cells, the VS1-, VS2- and VS3-cell. Because VS2 and VS3 have almost the same structure and very similar functional properties (Krapp et al., 1998), we did not distinguish between them. In each experiment, synaptic coupling between a VS-cell and V1 was confirmed by injecting current into the presynaptic neuron while monitoring the spike activity of V1. Depolarizing current increased the spike activity in V1, hyperpolarization led to a decrease in spike rate below the resting level. Relationships between the depolarizing presynaptic current and changes in postsynaptic spike rate are approximately linear (Fig. 1C). However, the slope of the relationship varied between preparations. Sometimes injection of only $+2 \mathrm{nA}$ led to more than 120 postsynaptic spikes/s, which is about half of the largest responses that are elicited in V1 by visual motion stimulation. In other experiments only smaller changes in postsynaptic spike rate could be elicited (Fig. 1C). This variability is not necessarily a consequence of variable synaptic gain, because the presynaptic depolarizations do not only depend on current strength, but also on methodological factors, such as the distance between the injection site and the presynaptic terminals.

\section{Synaptic transfer of slow presynaptic signals}

Despite differences in the resting potential of VS-cells, the relationship between the membrane potential changes elicited by band-limited white-noise velocity fluctuations and the corresponding instantaneous spike rate was basically the same for all analyzed cell pairs (Fig. 2). When the VS-cell hyperpolarized during upward motion, the spike rate of V1 did not change much. During downward motion the postsynaptic spike rate increased approximately lin- early with presynaptic depolarization for the entire activity range elicited by dynamic motion. A similar relationship was found for constant velocity motion (Kurtz et al., 2001). The gain between presynaptic potential and postsynaptic spike rate, as determined for the range between 50 and 200 postsynaptic spikes/s and the corresponding presynaptic membrane potentials, amounts to $17.9 \pm 3.8$ spikes/ $\mathrm{s} / \mathrm{mV}$ (mean \pm S.D.; 5 cell pairs).

To resolve synaptic transfer characteristics for the different frequencies in the presynaptic signal, a reverse reconstruction approach was applied (see Experimental Procedures). Usually this approach is employed to relate neuronal responses to sensory stimuli (for examples on fly motion vision see Bialek et al., 1991; Haag and Borst, 1997). In contrast, we utilized this method to determine to what extent the presynaptic potential fluctuations can be reconstructed by convolving the postsynaptic spike train with the linear filter that leads, on average, to the smallest error between the reconstructed and the recorded presynaptic signal. On a coarse timescale the graded potential fluctuations of the recorded and the reconstructed signals closely resemble each other, whereas on a fine timescale the presynaptic membrane potential cannot be recovered by reverse reconstruction (Fig. $3 \mathrm{~A}$ ).

The coherence function was determined to quantify to what extent the temporal frequency components of the presynaptic signal are linearly transformed into postsynaptic spike activity. For all analyzed cell pairs, the coherence between the reconstructed and the recorded presynaptic potential fluctuations is high $(>0.9)$ for temporal frequencies below approximately $10 \mathrm{~Hz}$. For higher frequencies the coherence decreases (Fig. 3B). Hence, for frequencies below $10 \mathrm{~Hz}$ the signal transfer between the presynaptic VS-cells and the postsynaptic V1-cell is approximately linear and reliable.

From the coherence function on its own it is not possible to assess whether signal transfer at frequencies above $10 \mathrm{~Hz}$ is unreliable or nonlinear (or both). Approaches established to disambiguate these two aspects (Haag and Borst, 1997; Roddey et al., 2000) cannot be applied to characterize the synaptic information transfer during visual stimulation, because they would require repeated trials in which the time course of the presynaptic potential were identical. This requirement, however, cannot be satisfied on the basis of experiments where synaptic signal transfer is investigated by visual stimulation, because of the large variability of the neuronal responses elicited by the same visual motion sequence. Therefore, a different approach was applied to characterize synaptic transfer of membrane potential fluctuations in a frequency range above $10 \mathrm{~Hz}$.

\section{Synaptic transfer of rapid presynaptic signals}

The spikelets that superimpose the graded depolarizations are the most pronounced rapid membrane potential changes found in the presynaptic neurons. Therefore, our analysis of synaptic signal transfer on a fine timescale focused on the relationship between these presynaptic 
A

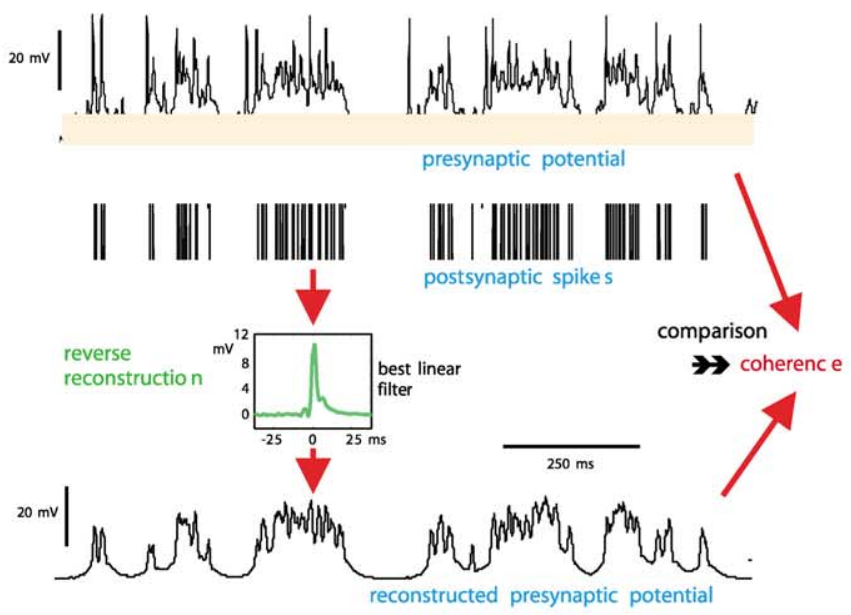

B

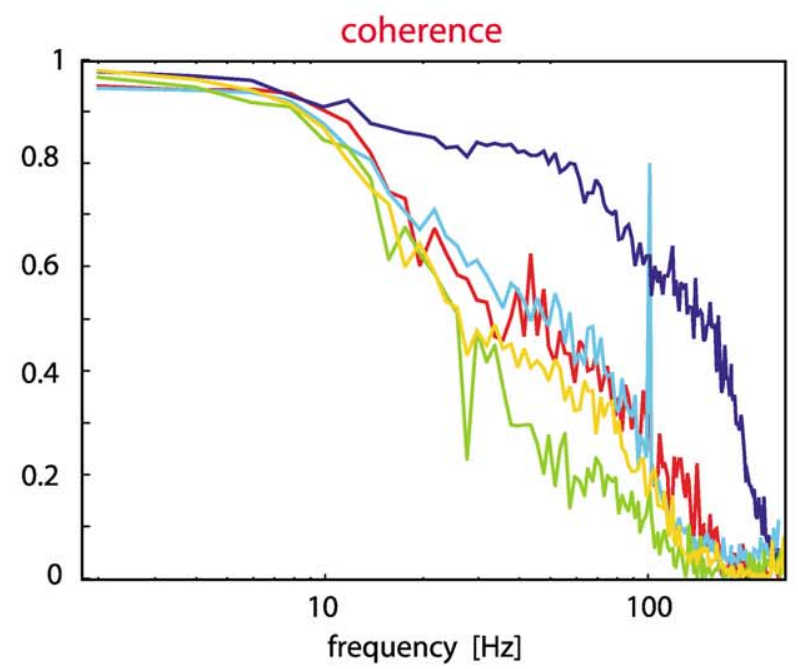

Fig. 3. Reverse reconstruction of the time-dependent presynaptic potential from postsynaptic spike trains. (A) Schematic outline of the procedure. The linear filter is determined which, when convolved with the postsynaptic spike train, leads to the best estimate of the presynaptic potential. Hyperpolarizations of the presynaptic neuron do not much affect the activity of the postsynaptic cell (due to the low postsynaptic resting activity). Therefore, the presynaptic potential was rectified at the resting potential of each response trace and was then used for the reconstruction. The resting potential was determined in a 1-s window before motion onset. The part of the response that was rectified is marked by the shaded bar. As a measure of the similarity between the recorded and the estimated presynaptic membrane potential traces the coherence function was determined. (B) Coherence determined for the five cell pairs analyzed with random-velocity fluctuations. Cell pairs are the same as in Fig. 2 . The coherence values close to 1 for frequencies up to $10 \mathrm{~Hz}$ indicate that the system can be regarded as very reliable and approximately linear in this frequency range.

signal components and the timing of the postsynaptic spikes.

The average presynaptic potential that coincides during dynamic motion stimulation with a postsynaptic spike consists of a depolarization with a width at half-maximum amplitude of some tens of milliseconds that is superimposed by a peak of only a few milliseconds width (Fig. 4A, left). This peak indicates that not only relatively slow depolarizations are synaptically transmitted, but also spikelets. The shuffled spike-triggered averages that were elicited by the same motion sequence, but not recorded simultaneously, only show the broad but not the narrow peak (Fig. 4A, right). Hence, the spikelets do not appear to be tightly time locked to stimulus motion (see also below).

The same conclusion can be drawn for all analyzed cell pairs from the frequent coincidences of fast presynaptic membrane potential transients, such as spikelets, and postsynaptic spikes. Since presynaptic spikelets do not have a constant amplitude but vary considerably in size, there is no generally applicable criterion to isolate spikelets. In order to investigate the effects of rapid membrane potential transients we decided to select depolarizations with a slope of more than $4 \mathrm{mV} / \mathrm{ms}$. By this criterion, rapid membrane potential transients - in particular most of the spikelets-are included in the analysis. The reliability of transmission of these presynaptic membrane potential transients was quantified by determining the relative frequency of presynaptic potential transients that lead to a postsynaptic spike within time bins of $0.24 \mathrm{~ms}$ (Fig. 4B, left). A large proportion $(0.67 \pm 0.18$; mean $\pm S$.D. for the analyzed cell pairs) of presynaptic depolarizing transients cause a postsynaptic spike within a time window of \pm 0.96 ms. Again, the narrow correlation peaks disappear when coincidences between presynaptic depolarization transients and postsynaptic spikes are determined in traces that were evoked by the same stimulus, but not recorded simultaneously (Fig. 4B, right). If presynaptic spikelets and postsynaptic spikes would time lock with a millisecond precision to dynamic motion stimuli, the shuffled correlograms should show a similarly narrow peak as was found for simultaneously recorded responses. Consequently, VS-V1 synapses transmit a large proportion of rapid depolarizations to the postsynaptic cell, although many of these membrane potential transients are not precisely time locked to the visual stimulus.

Reliable transmission of rapid depolarisations was further corroborated in another way. Here the reliability of synaptic signal transfer was quantified by relating the S.D. of the timing of the first postsynaptic spike after the onset of constant velocity motion to the corresponding temporal change in presynaptic depolarization. We analyzed the onset of a motion stimulus because this represents the most rapid velocity change that is conceivable. This stimulation procedure is chosen to investigate the system at its limits. For three pattern contrasts the slope of the presynaptic potential was determined for the initial response peak after motion onset. To account for response latencies (Warzecha and Egelhaaf, 2000), the first spike which was generated later than $20 \mathrm{~ms}$ after motion onset was used for determining the precision of spike timing. The spike jitter decreases monotonically with increasing slope of the presynaptic potential. It may decrease to only a few milliseconds when the presynaptic membrane potential depolarizes very fast as is characteristic of the motion onset of a 

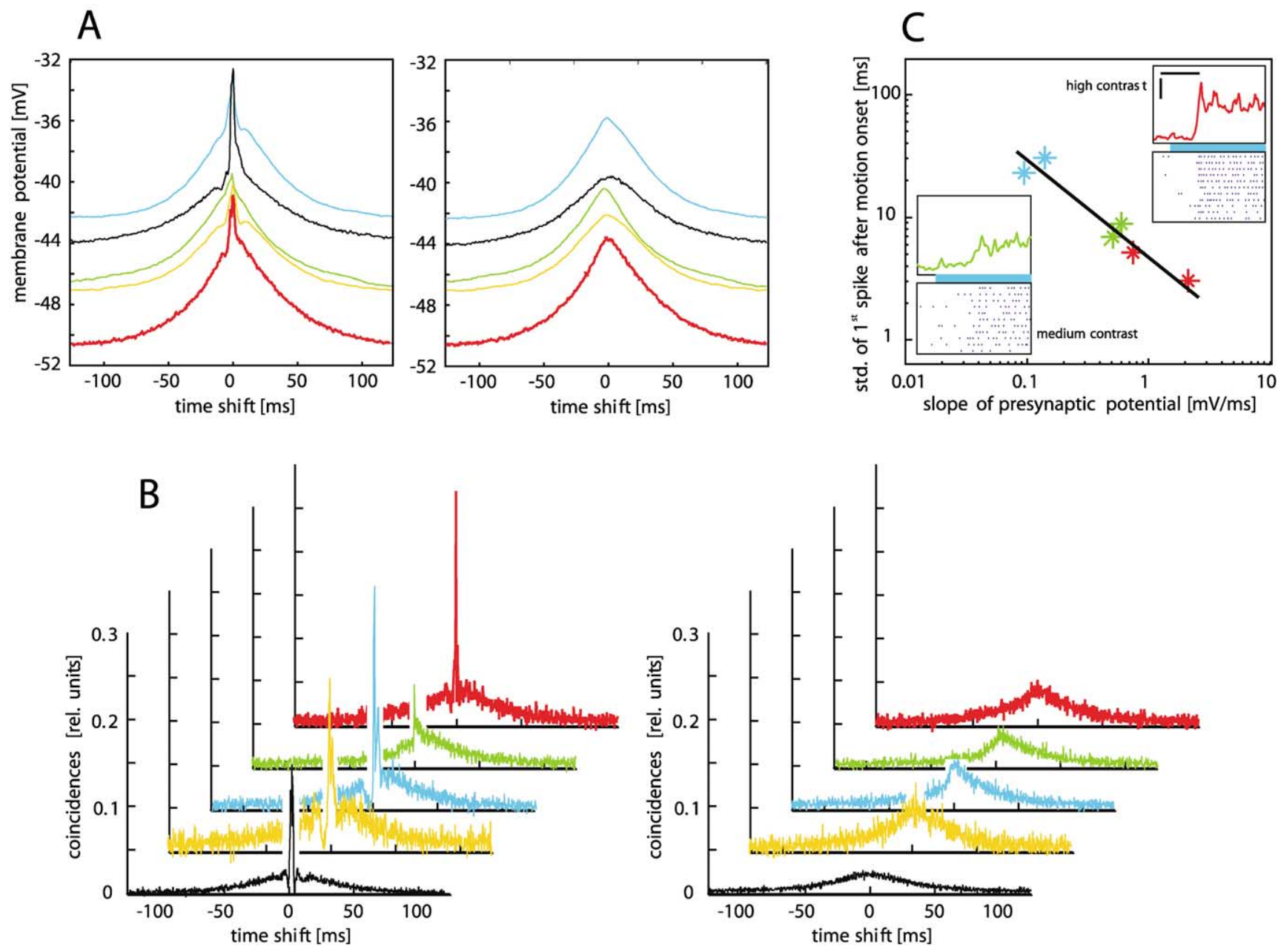

Fig. 4. Relationship between pre- and postsynaptic responses on a fine timescale. (A) Average presynaptic potential that elicits a postsynaptic spike as determined from cross-correlating the responses of a VS-cell with the spike trains of the V1-cell. Either the simultaneously recorded responses (left) or the responses that were elicited by the same motion sequence, but not recorded simultaneously (right), were cross-correlated. All responses during dynamic motion stimulation were used for the analysis irrespective of the instantaneous stimulus velocity. The analysis was done for five cell pairs separately as indicated by the different colors. (B) Coincidences between presynaptic membrane potential transients and postsynaptic spikes of simultaneously recorded responses (left) and responses that were elicited by the same stimulus but not recorded simultaneously (right). Depolarizations with a slope of larger than $4 \mathrm{mV} / \mathrm{ms}$ were selected as membrane potential transients. The analysis was conducted at a temporal resolution of $0.24 \mathrm{~ms}$. The number of coincidences was normalized with respect to the number of presynaptic potential transients. Hence an ordinate value of 0.3 indicates that $30 \%$ of the presynaptic potential transients elicit a postsynaptic spike within $0.24 \mathrm{~ms}$. The analysis was done with the same five cell pairs as used in A. (C) Relationship between changes in presynaptic membrane potential and the temporal jitter in the occurrence of the first postsynaptic spike after motion onset. Three pattern contrasts $(0.14,0.26$ and 0.67$)$ were used to vary the stimulus strength. The slope of the presynaptic potential was determined as the ratio between the time it took the cell to depolarize to the initial response peak after motion onset starting at the $30 \%$ peak level and the corresponding membrane potential values. To account for response latencies, the first spike occurring at least $20 \mathrm{~ms}$ after motion onset was used for determining the precision of spike timing. The jitter in spike timing was determined as the across-trial S.D. of the times of occurrence of the first spike. The insets give two examples of averaged presynaptic potentials (upper diagrams) and raster plots of sequences of individual spike responses (lower diagrams). The blue horizontal bar indicates the time of motion stimulation. The timescale of the insets is given in the upper right diagram, it denotes $50 \mathrm{~ms}$. The vertical scale bar indicates $5 \mathrm{mV}$. The data are based on two dual recordings in each of which all contrasts were tested.

high-contrast pattern. The spike jitter is much larger when the slope of the presynaptic potential is only shallow, as is characteristic of motion onsets of low-contrast stimuli (Fig. 4C).

These results suggest that synaptic signal transfer between VS-cells and V1 can be very precise on a fine timescale provided that the presynaptic membrane potential depolarizes rapidly. This conclusion raises the question of on what timescale information about visual motion is transmitted at VS-V1 synapses.

\section{Relationship between synaptic signal transfer and motion stimuli}

The relationship between visual motion stimuli and the performance of synaptic signal transfer was assessed in several ways. It was concluded above that, in accordance with previous findings (Warzecha et al., 1998), most spikes and spikelets in fly TCs do not time lock with a millisecond precision to dynamic motion stimuli (Fig. 4A, B). Fast mem- 
A

motion-induced response / motion stimulus

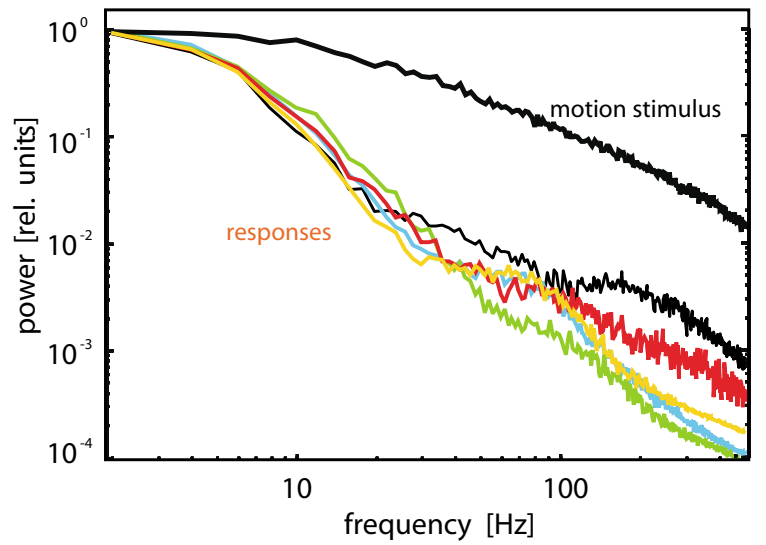

B

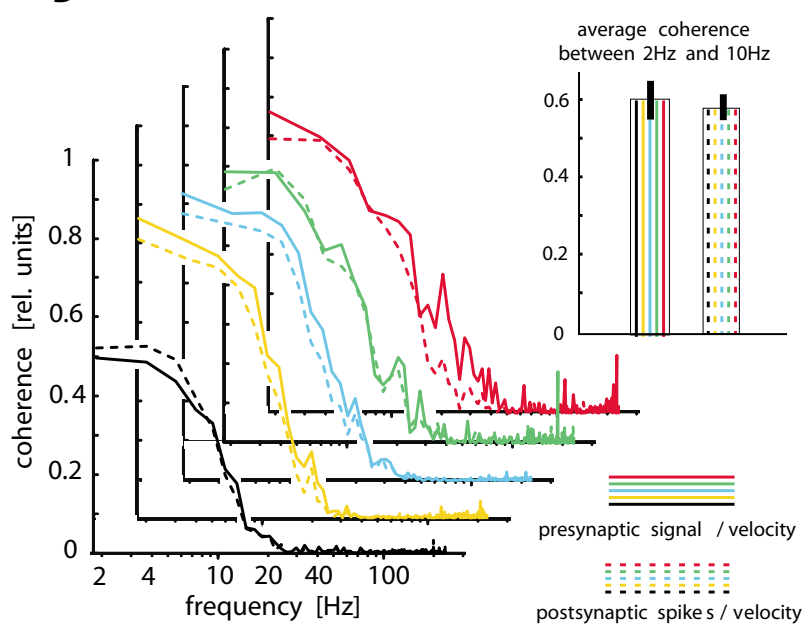

Fig. 5. Relation between motion stimuli and neuronal responses. The data obtained with the five analyzed cell pairs are shown. (A) Power spectra of the motion stimulus, i.e. the random-velocity fluctuations, and of the stimulus-induced response component of the presynaptic VS-cell, i.e. the component of the stimulus that is directly linked to the stimulus and free of noise. For each cell pair, the power spectrum is the average obtained from the responses to all 16 stimulus traces. (B) Reverse reconstruction of the stimulus from both the pre- and the postsynaptic signal. To account for the rectification nonlinearity due to spike generation and the low spontaneous activity of $\mathrm{V} 1$, the presynaptic membrane potential traces and the velocity traces were rectified. The threshold for the presynaptic potential was the resting potential as determined during $1 \mathrm{~s}$ before motion onset. During null-direction motion the velocity of the stimulus trace was set to zero. The relationship between the stimulus and the neuronal response is quantified by the coherence functions. The mean coherences between frequencies of $2 \mathrm{~Hz}$ and $10 \mathrm{~Hz}$ averaged over the five cell pairs are shown by the inset together with the corresponding S.D.S.

brane potential depolarizations are needed to trigger spikes with a high temporal precision (Mainen and Sejnowski, 1995; Nowak et al., 1997; Kretzberg et al., 2001). However, as a consequence of the mechanism of visual motion computation, the neural representation of highfrequency velocity fluctuations is largely attenuated (Egelhaaf and Reichardt, 1987; Haag and Borst, 1997; Warzecha et al., 1998). This feature of visual motion computation also becomes obvious when analyzing power spectra of the stimulus-induced component of VS-cell responses (Fig. 5A). At low frequencies the power of the membrane potential fluctuations is large, but it decreases with increasing frequencies much more steeply than the power of the velocity fluctuations of the motion stimulus does. Thus, for frequencies up to about $10 \mathrm{~Hz}$, where velocity fluctuations are transmitted with a high gain, the signal transfer at VS-V1 synapses is almost linear (compare Figs. $3 B$ and $5 A$ ).

As a consequence of the reliability of VS-V1 synapses and the linearity of signal transfer in the low-frequency range, preferred-direction motion can be estimated by reverse reconstruction almost equally well from presynaptic and from postsynaptic signals (Fig. 5B). The average coherence between sections of the stimulus with motion in the preferred direction and the corresponding neuronal response sections is only insignificantly smaller when the postsynaptic response rather than the presynaptic response is taken into account. In accordance with previous findings (Haag and Borst, 1997), this similarity also suggests that different response modes, i.e. graded versus spiking, do not per se affect the reliability with which motion information is encoded. Since the relationship between stimulus velocity and the time course of the TCs' response is highly nonlinear for rapidly varying motion stimuli (Egelhaaf and Reichardt, 1987), the relatively low coherence can be attributed partly to the mechanisms underlying visual motion processing (Haag and Borst, 1997). Notwithstanding, the relationship between stimulus and neuronal response is-apart from rectification-not much affected by the intervening synapse and by spike generation in the postsynaptic neuron.

\section{DISCUSSION}

Synaptic signal transfer was analyzed for pairs of identified motion-sensitive neurons of the fly, stimulated in a natural way by sensory input rather than artificially by current injection. In this way the range of presynaptic membrane potential amplitudes as well as the timescale on which the presynaptic potential varied came close to the natural operating range of the system. It was shown that both graded membrane potential changes and rapid spike-like depolarizations are transmitted. Transfer of visual motion information is linear and reliable not only under steady-state conditions (Kurtz et al., 2001), but also for presynaptic membrane potential fluctuations up to approximately $10 \mathrm{~Hz}$. Furthermore, rapid presynaptic potential changes, such as spikelets, elicit postsynaptic spikes with a high probability, although most of the rapid potential changes are not timelocked precisely to visual motion stimulation (Fig. 4A, B; see also Warzecha et al., 1998). Precise time locking of neuronal signals to motion stimuli occurs in fly TCs only after very rapid velocity changes (de Ruyter van Steveninck et al., 2001; de Ruyter van Steveninck and Bialek, 1988, 1995; Warzecha and Egelhaaf, 2001). This 
high degree of precision is preserved at VS-V1 synapses (Fig. 4C).

The V1-cell receives its input from three VS-cells. The receptive fields of all three input elements overlap but are slightly displaced with respect to each other (Krapp et al., 1998). Although no dual recordings from VS-cells are currently available, the temporal fine structure of VS-cell responses is likely to be highly correlated. This is expected, because for other fly TCs with overlapping receptive fields and, thus, largely common synaptic input, spikes tend to synchronize with a millisecond precision. Such synchrony occurs despite of the fact that most of the spikes are not time locked to the visual motion stimuli (Warzecha et al., 1998). This feature needs to be kept in mind when interpreting the high correlation between rapid presynaptic depolarisations and postsynaptic spikes. By visual stimulation it is not possible to activate only one of the VS-cells exclusively because their receptive fields overlap to a large extent. Therefore, an analysis based on visual stimulation, like ours, does not allow definite conclusions about the transmission properties of a single synapse. Moreover, it is not easily possible to ablate individual presynaptic VS-cells to study the influence of visually elicited activity of the remaining ones on the postsynaptic responses. Despite these qualifications, the current analysis allows us to assess the impact of synaptic transfer on the encoding of the natural sensory input of the system. Linearity and reliability of signal transfer are functionally relevant, regardless of the synaptic mechanisms which provide them.

\section{Signal transfer at synapses on different timescales}

Linear signal transfer between neurons cannot be taken for granted, since the overall synaptic transmission properties as well as the underlying cellular processes were found to be highly nonlinear in many systems (e.g. Johnston and Wu, 1995; Augustine, 2001). Synaptic transfer between spiking neurons was found to be sigmoidal after blocking the sodium channels by TTX (Katz and Miledi, 1966; Graubard and Calvin, 1979). Similar sigmoidal synaptic transfer characteristics were reported for graded synaptic transmission (e.g. Simmons, 1999). In contrast to synaptic transmission of spikes, the threshold for transmitter release at graded synapses is frequently close to the resting potential and thus also allows for the transmission of small changes in presynaptic membrane potential (Burrows and Siegler, 1978; Graubard and Calvin, 1979; see also Fig. 2). For the analyzed VS-V1 synapses a linear relationship between presynaptic membrane potential and postsynaptic spike rate was found for the entire range of presynaptic depolarizations elicited by preferred-direction motion. The saturation range of a sigmoidal synaptic transmission characteristic is not reached even by very strong visual stimuli. In contrast, null-direction motion which hyperpolarizes the presynaptic VS-cell below its resting potential does not much affect postsynaptic activity (Fig. 2; see also Kurtz et al., 2001).

Not only relatively slow graded membrane potential changes are transmitted at VS-V1 synapses, but also rapid spike-like presynaptic depolarizations. This finding suggests that synaptic transmission is not limited to the relatively slow changes of the graded presynaptic membrane potential but operates on different timescales. A similar conclusion was drawn for the stomatogastric system of crustaceans. Here the amplitude of the postsynaptic response was found to depend on the time course of the presynaptic depolarization waveform. Transmitter release was found to be larger when evoked by graded membrane potential changes than when evoked by action potentials (e.g. Manor et al., 1997). In the peripheral visual system of locusts synaptic transmission of graded potentials depends on the dynamic properties of the presynaptic signal (Simmons, 2002a). In this study it could be shown that the rate at which the presynaptic potential depolarizes determines the resulting membrane potential change in the postsynaptic neuron. These features of synaptic transmission may be highly relevant when the presynaptic potential changes at a wide range of timescales, as is the case in VS-cells during visual motion stimulation. However, in contrast to the synapses of the locust system which owe their characteristic transmission properties to synaptic depression (Simmons, 2002a), there is no indication of depression or facilitation at fly VS-V1 synapses. For instance, even during prolonged motion stimulation the presynaptic potential and the postsynaptic spike rate are largely proportional (Warzecha, unpublished observations). Moreover, when the linear reconstruction method is used to recover the presynaptic potential from the postsynaptic spike trains, the linear filters determined for the first second of dynamic motion stimulation did not differ consistently from those determined for the last second of stimulation (Warzecha, unpublished observations). Current experiments in which the membrane potential and/or presynaptic calcium of VS-cells are manipulated in a well-defined way by either current injection or flash photolysis of caged calcium are aimed at unraveling the synaptic transfer more systematically than is possible with natural sensory stimulation (Kurtz, unpublished observations).

There is evidence that transmitter release in spiking and graded-potential neurons is caused by different calcium currents. In leech heart interneurons graded transmission was found to be mediated by low-threshold calcium currents (Angstadt and Calabrese, 1993; Ivanov and Calabrese, 2000), whereas high-threshold calcium currents appear to underlie spike-mediated synaptic transmission (Lu et al., 1997; Ivanov and Calabrese, 2000). It needs to be analyzed in future experiments whether equivalent mechanisms underlie signal transmission at slow and fast timescales at VS-V1 connections.

Although there are many studies on the reliability of synaptic transfer in spiking neurons (review: Faber et al., 1998), there are only few studies, mainly in the peripheral insect visual system, on the reliability of graded synapses (Laughlin et al., 1987; Juusola et al., 1995; De Ruyter van Steveninck and Laughlin, 1996; Simmons, 1999, 2002b). In the latter systems synaptic transmission was found to be a major cause of variability in the postsynaptic potential limiting the smallest size of change in presynaptic potential that can be transmitted faithfully across the synapse. In 
contrast, most of the noise limiting the reliability of the system analyzed in the present account appears to originate peripherally to the investigated synapse because the velocity of the motion stimulus can be reconstructed almost equally well by a linear system on the basis of the pre- and postsynaptic signals (Fig. 5).

\section{Synaptic performance in the behavioral context}

The response amplitude of all TCs depends on a variety of stimulus parameters, most notably on stimulus velocity (e.g. Hausen, 1981; Hausen and Egelhaaf, 1989). TCs are tuned to particular types of optic flow as it is induced on the eyes during self-motion (Egelhaaf et al., 2002; Hausen and Egelhaaf, 1989). The neuronal computation of self-motion is facilitated by a comparison of visual motion information originating from both eyes (reviews: Hausen, 1981; Dahmen et al., 2000; Egelhaaf et al., 2002; Borst and Haag, 2002). If an element relays motion information linearly from one side of the brain to the other, like the V1-neuron, the stimulus-dependence of the neuronal activity is maintained. Otherwise the dependence on stimulus parameters would be different for heterolateral and unilateral TCs and could impede a comparison of visual motion information from both eyes. Linear synaptic signal transfer may thus be adaptive in the functional context of self-motion estimation on the basis of binocular optic flow.

Signal transfer between VS-V1 neurons is linear for graded presynaptic membrane potential fluctuations of up to approximately $10 \mathrm{~Hz}$. Interestingly, this range fits to the range in which visual motion is transmitted with a high gain by the visual system. As a consequence of the mechanisms underlying visual motion computation, velocity fluctuations at frequencies above $10 \mathrm{~Hz}$ are represented by TCs with decreasing gain. Above approximately $20 \mathrm{~Hz}$ the membrane potential noise in TCs was found to exceed the stimulus-induced response component (Haag and Borst, 1997; Warzecha et al., 1998). As a consequence, the timing of spikes on a millisecond timescale is mainly determined by noise and not by velocity fluctuations. These findings imply that VS-V1 synapses operate approximately linearly in a frequency range where the visual motion pathway is most sensitive to changes in velocity. The significance of all these features of TCs and, in particular, of the synaptic signal transfer of the VS-V1 synapses for the operating conditions during normal behavior needs to be assessed with stimuli that are not designed by the experimenter but are generated by the behaving animal by its actions and reactions. Thanks to recent advances of technology this sort of analysis is now possible (Egelhaaf et al., 2002; Lindemann et al., 2002; Kern et al., 2001). Notwithstanding, our current systems analysis based on visual motion stimuli that were specially designed by the experimenter sets the conceptual frame which is necessary to understand the responses to the much more complex behaviorally generated visual motion stimuli.

Acknowledgements-We are grateful to N. Böddeker, J. Grewe, K. Karmeier, R. Kern and C. Petereit for helpful comments on the manuscript. Supported by the Deutsche Forschungsgemeinschaft (DFG).

\section{REFERENCES}

Angstadt JD, Calabrese RL (1993) Calcium currents and graded synaptic transmission between heart interneurons of the leech. J Neurosci 11:746-759.

Augustine GJ (2001) How does calcium trigger neurotransmitter release? Curr Opin Neurobiol 11:320-326.

Bialek W, Rieke F, de Ruyter van Steveninck R, Warland D (1991) Reading a neural code. Science 252:1854-1857.

Borst A, Haag J (2002) Neural networks in the cockpit of the fly. J Comp Physiol A 188:419-437.

Borst A, Theunissen FE (1999) Information theory and neural coding. Nat Neurosci 2:947-957.

Burrows M, Siegler MVS (1978) Graded synaptic transmission between local interneurones and motoneurones in the metathoracic ganglion of the locust. J Physiol 285:231-255.

Dahmen HJ, Franz MO, Krapp HG (2000) Extracting ego-motion from optic flow: limits of accuracy and neuronal filters. In: Computational, neural and ecological constraints of visual motion processing (Zanker JM, Zeil J, eds), pp 143-168. Berlin: Springer.

Dayan P, Abbott LF (2001) Theoretical neuroscience: computational and mathematical modeling of neural systems. MIT Press: Cambridge, MA.

de Ruyter van Steveninck R, Bialek W (1988) Real-time performance of a movement-sensitive neuron in the blowfly visual system: coding and information transfer in short spike sequences. Proc R Soc Lond B 234:379-414.

de Ruyter van Steveninck R, Bialek W (1995) Reliability and statistical efficiency of a blowfly movement-sensitive neuron. Phil Trans R Soc Lond B 348:321-340.

de Ruyter van Steveninck R, Borst A, Bialek W (2001) Real-time encoding of motion: answerable questions and questionable answers from the fly's visual system. In: Motion vision (Zanker JM, Zeil J, eds), pp 279-306. Berlin: Springer.

de Ruyter van Steveninck R, Laughlin SB (1996) The rate of information transfer at graded-potential synapses. Nature 379:642-645.

Dürr V, Egelhaaf M (1999) In vivo calcium accumulation in presynaptic and postsynaptic dendrites of visual interneurons. J Neurophysiol 82:3327-3338

Egelhaaf M, Kern R, Krapp HG, Kurtz R, Warzecha A-K (2002) Neural encoding of behaviourally relevant motion information in the fly. Trends Neurosci 25:96-102.

Egelhaaf M, Reichardt W (1987) Dynamic response properties of movement detectors: theoretical analysis and electrophysiological investigation in the visual system of the fly. Biol Cybern 56:69-87.

Faber DS, Korn H, Redman SJ, Thompson SM, Altman JSE (1998) Central synapses: quantal mechanisms and plasticity. Strasbourg: HFSP.

Fortune ES, Rose GJ (1997) Passive and active membrane properties contribute to the temporal filtering properties of midbrain neurons in vivo. J Neurosci 17:3815-3825.

Fortune ES, Rose GJ (2001) Short-term synaptic plasticity as a temporal filter. Trends Neurosci 24:381-385.

Gabbiani F, Koch C (1998) Principles of spike train analysis. In: Methods in neuronal modeling (Koch C, Segev I, eds), pp 313-360. Cambridge, MA: MIT Press.

Graubard K, Calvin WH (1979) Presynaptic dendrites: implications of spikeless synaptic transmission and dendritic geometry. In: The neurosciences: fourth study program (Schmitt FO, Worden FG, eds), pp 317-331. Cambridge, MA: MIT Press.

Haag J, Borst A (1997) Encoding of visual motion information and reliability in spiking and graded potential neurons. J Neurosci 17 : 4809-4819.

Haag J, Borst A (2001) Recurrent network interactions underlying 
flow-field selectivity of visual interneurons. J Neurosci 21:56855692.

Haag J, Theunissen F, Borst A (1997) The intrinsic electrophysiological characteristics of fly lobula plate tangential cells: II. Active membrane properties. J Comput Neurosci 4:349-369.

Hausen K (1981) Monocular and binocular computation of motion in the lobula plate of the fly. Verh Dtsch Zool Ges 74:49-70.

Hausen K (1982) Motion sensitive interneurons in the optomotor system of the fly: I. The horizontal cells: structure and signals. Biol Cybern 45:143-156.

Hausen K, Egelhaaf M (1989) Neural mechanisms of visual course control in insects In: Facets of vision (Stavenga D, Hardie RC, eds), pp 391-424. Berlin: Springer.

Hengstenberg R (1982) Common visual response properties of giant vertical cells in the lobula plate of the blowfly Calliphora. J Comp Physiol 149:179-193.

Horstmann W, Egelhaaf M, Warzecha A-K (2000) Synaptic interactions increase optic flow specificity. Eur J Neurosci 12:2157-2165.

Isaacson JS, Strowbridge BW (1998) Olfactory reciprocal synapses: dendritic signaling in the CNS. Neuron 20:749-761.

Ivanov Al, Calabrese RL (2000) Intracellular $\mathrm{Ca}^{2+}$ dynamics during spontaneous and evoked activity of leech heart interneurons: lowthreshold $\mathrm{Ca}$ currents and graded synaptic transmission. J Neurosci 20:4930-4943.

Johnston D, Wu M-S (1995) Foundations of cellular neurophysiology. Cambridge, MA: MIT Press.

Juusola M, Uusitalo RO, Weckström M (1995) Transfer of graded potentials at the photoreceptor-interneuron synapse. J Gen Physiol 103:117-148.

Katz B, Miledi R (1966) Input-output relation of a single synapse. Nature 212:1242-1245.

Kern R, Petereit C, Egelhaaf M (2001) Neural processing of naturalistic optic flow. J Neurosci 21:1-5.

Krapp HG, Hengstenberg B, Hengstenberg R (1998) Dendritic structure and receptive-field organization of optic flow processing interneurons in the fly. J Neurophysiol 79:1902-1917.

Krapp HG, Hengstenberg R, Egelhaaf M (2001) Binocular contribution to optic flow processing in the fly visual system. J Neurophysiol 85:724-734.

Kretzberg J, Egelhaaf M, Warzecha A-K (2001) Membrane potential fluctuations determine the precision of spike timing and synchronous activity: a model study. J Comput Neurosci 10:79-97.

Kurtz R, Warzecha A-K, Egelhaaf M (2001) Transfer of visual information via graded synapses operates linearly in the natural activity range. J Neurosci 21:6957-6966.

Laughlin SB, Howard J, Blakeslee B (1987) Synaptic limitations to contrast coding in the retina of the blowfly Calliphora. Proc R Soc Lond B 231:437-467.

Lindemann JP, Kern R, Meyer P, van Hateren JH, Egelhaaf M (2002) FliMax, a novel stimulus device for panoramic and high-speed presentation of behaviourally generated optic flow. Vision Res 43: 779-791.
Lu J, Dalton F, Stokes DR, Calabrese RL (1997) Functional role of calcium currents in graded and spike-mediated synaptic transmission between leech heart interneurons. J Neurophysiol 77:1779_ 1794.

Machens CK, Stemmler MG, Prinz P, Krahe R, Ronacher B, Herz AVM (2001) Representation of acoustic communication signals by insect auditory receptor neurons. J Neurosci 21:3215-3227.

Mainen ZF, Sejnowski TJ (1995) Reliability of spike timing in neocortical neurons. Science 268:1503-1506.

Manor Y, Nadim F, Abbott LF, Marder E (1997) Temporal dynamics of graded synaptic transmission in the lobster stomatogastric ganglion. J Neurosci 17:5610-5621.

Marmarelis PZ, Marmarelis VZ (1978) Analysis of physiological systems. Plenum Press: New York.

Nowak LG, Sanchez-Vives MV, McCormick DA (1997) Influence of low and high frequency inputs on spike timing in visual cortical neurons. Cereb Cortex 7:487-501.

Roddey JC, Girish B, Miller JP (2000) Assessing the performance of neural encoding models in the presence of noise. $\mathrm{J}$ Comput Neurosci 8:95-112.

Simmons PJ (1999) The performance of synapses that convey discrete graded potentials in an insect visual pathway. J Neurosci 19:10584-10594.

Simmons PJ (2002a) Presynaptic depolarization rate controls transmission at an invertebrate synapse. Neuron 35:749-758.

Simmons PJ (2002b) Signal processing in a simple visual system: the locust ocellar system and its synapses. Microsc Res Tech 56:270 280.

Strausfeld NJ, Kong A, Milde JJ, Gibert C, Ramaiah L (1995) Oculomotor control in calliphorid flies: GABAergic organization in heterolateral inhibitory pathways. J Comp Neurol 361:298-320.

Thomson AM (2000) Facilitation, augmentation and potentiation at central synapses. Trends Neurosci 23:305-312.

Trussell LO (1999) Synaptic mechanisms for coding timing in auditory neurons. Annu Rev Neurosci 61:477-496.

Warzecha A-K, Egelhaaf M (1999) Variability in spike trains during constant and dynamic stimulation. Science 283:1927-1930.

Warzecha A-K, Egelhaaf M (2000) Response latency of a motionsensitive neuron in the fly visual system: dependence on stimulus parameters and physiological conditions. Vision Res 40:29732983.

Warzecha A-K, Egelhaaf M (2001) Neuronal encoding of visual motion in real-time. In: Processing visual motion in the real world: a survey of computational, neural, and ecological constraints (Zanker JM, Zeil J, eds), pp 239-277. Berlin: Springer.

Warzecha AK, Egelhaaf M, Borst A (1993) Neural circuit tuning fly visual interneurons to motion of small objects. I. Dissection of the circuit by pharmacological and photoinactivation techniques. J Neurophysiol 69:329-339.

Warzecha A-K, Kretzberg J, Egelhaaf M (1998) Temporal precision of the encoding of motion information by visual interneurons. Curr Bio 8:359-368. 